\title{
Combined analysis of genomewide scans for adult height: results from the NHLBI Family Blood Pressure Program
}

\author{
Xiaodong $\mathrm{Wu}^{1}$, Richard S. Cooper ${ }^{1}$, Eric Boerwinkle ${ }^{2}$, Stephen T. Turner ${ }^{3}$, Steve Hunt ${ }^{4}$, \\ Richard Myers ${ }^{5}$, Richard A Olshen ${ }^{6}$, David Curb ${ }^{7}$, Xiaofeng Zhu ${ }^{1}$, Donghui Kan ${ }^{1}$ and \\ Amy Luke ${ }^{1}$
}

\begin{abstract}
${ }^{1}$ Department of Preventive Medicine and Epidemiology, Loyola University Medical Center, Maywood, IL, USA; ${ }^{2}$ Institute for Molecular Medicine and Human Genetics Center, University of Texas Houston Health Science Center, Houston, TX, USA; ${ }^{3}$ Division of Hypertension, Mayo Clinic, Rochester, MN, USA; ${ }^{4}$ Cardiovascular Genetics Research Clinic, University of Utah School of Medicine, Salt Lake City, UT, USA; ${ }^{5}$ Section of Neurogenetics, Boston University Medical Center, Boston, MA, USA; ${ }^{6}$ Department of Health Research and Policy, Stanford University School of Medicine, Stanford, CA, USA; ${ }^{7}$ Pacific Health Research Institute, Honolulu, HI, USA
\end{abstract}

A combined analysis of genome scans was performed for adult height in the NHLBI Family Blood Pressure Program. Height data were available on 6752 individuals. Linkage analysis was performed first separately for each of the eight ethnic groups in the four networks using the variance component method. To increase the power to detect the common genetic components affecting height for all the individuals, a linkage analysis was performed subsequently for the combined data set by pooling the average allelesharing IBD () for all groups. By combining the data, we replicated evidence for a QTL influencing adult height on chromosome $7(7 q 31)(L O D=2.46)$, which has been reported in two previous studies. Suggestive linkage (LOD $>1$ ) was found in another six regions in our combined analysis. Evidence for linkage for two of these regions $(2 \mathrm{p} 12,20 \mathrm{p} 11)$ has also been reported previously. European Journal of Human Genetics (2003) 11, 271-274. doi:10.1038/sj.ejhg.5200952

Keywords: height; BMl; genome scan; linkage

\section{Introduction}

Adult height is a complex genetic trait with high heritability (>80\%). ${ }^{1-5}$ Genetic studies of adult height have revealed that it is normally distributed, suggesting that height could be affected by the interaction of multiple genetic and environmental factors. Compared with other complex traits, adult height can be measured easily and accurately, reducing error variance in the phenotype. In

*Correspondence: $\mathrm{Dr} \mathrm{X}$ Wu, Department of Preventive Medicine and Epidemiology, Loyola University Medical Center, 2160 S. First Ave, Maywood, IL 60153, USA. Tel: +1 708327 9070; Fax: +1 708327 9009; E-mail: xwu@apache.medctr.luhs.org

Received 14 May 2002; revised 22 October 2002; accepted 19 December 2002 most genetic studies for other complex human traits, height has been collected along with other data. Obviously, this raises the possibility of pooling data. Several recent genome scans for adult height revealed significant and suggestive evidence for linkage on several chromosomes. ${ }^{4-7}$ However, few of these regions were replicated among different studies. This may not be surprising considering that height is a complex genetic trait affected by multiple genes, each having a small effect, as well as by environmental factors. An obvious step to increase power and decrease false-positive results in linkage analysis of complex traits is to pool data across studies, so long as one is careful regarding the heterogeneity among different data sets. ${ }^{8,9}$ Here we report the results of our combined analysis of genome scans for adult height in the ongoing NHLBI 
Family Blood Pressure Program (FBPP). We first performed the genome scan for adult height within each ethnic group of the four networks in FBPP separately. We then performed a genome scan using information from all the groups by combining IBD sharing estimated from each group separately.

\section{Materials and methods}

Subjects were selected from the four component networks (GenNet, GENOA, HyperGEN, and SAPPHIRe) of the FBPP. All the families were ascertained by hypertensive probands. The ascertainment scheme and program design have been reported elsewhere. ${ }^{10}$ In brief, GenNet sampled African-American and European-American nuclear families through identification of a young-middle-aged proband with elevated blood pressure (BP). Both GENOA and HyperGEN sampled African-American and European-American sibships containing sibpairs with essential hypertension. GENOA also sampled Mexican-American sibships containing sibpairs with hypertension, as well as some non hypertensive sibs in all three ethnic groups. SAPPHIRe recruited groups of Japanese and Chinese sibpairs that were concordant for hypertension or extremely discordant. In each network, we combined the data according to ethnic background, including European Americans and African Americans from GenNet; European Americans, African Americans and Mexican Americans from GENOA; European Americans and African Americans from HyperGEN; and Asians from SAPPHIRe.

DNA was extracted from whole blood by standard methods at each of the four networks and was sent to the Mammalian Genotyping Service in Marshfield, WI for analysis. Screening Set 8 (372 highly polymorphic microsatellite markers) was used for all four networks. This screening set has an average heterozygosity of $80 \%$, an average inter-marker distance of $10 \mathrm{~cm}$, and covers $95 \%$ of the human genome. Some aspects of the marker data upon which this paper is based are under review by members of the FBPP group. However, no edits of the data contem- plated thus far have any bearing on the inferences of this paper.

Individuals younger than 20 years old were excluded from the analysis because growth may not have reached a maximum. Height was adjusted by age for each gender separately in each of the eight groups and the residuals were standardized to obtain $Z$ scores. These were the phenotypes in our analyses. Heritability for height was estimated for each group separately using the variance component method. The genome scans were performed using the multipoint variance component method in SOLAR. ${ }^{11}$ The VC method specifies the expected genetic covariances between relatives as a function of the estimated proportion of alleles sharing IBD at a marker locus. The IBD probabilities were estimated using a multipoint approach that considers all available genotypes. The likelihood ratio test was applied to test the null hypothesis of no additive genetic variance due to a quantitative trait locus (QTL) at a particular location. To obtain the empiric genomewide significance of this result, we performed simulations by randomly generating genotypes while retaining the phenotypes and pedigrees under the hypothesis of no linkage. Maximum LOD score (MLS) from each simulation was recorded and the $P$-value for our genome scan was obtained by calculating the frequency of MLS larger than the tested LOD score.

It is well known that allele frequencies between different human populations can be quite different. The estimates of allele frequencies could be biased if we used the combined data set directly; in turn, this could bias the estimates of IBD. To get an unbiased estimate of IBD, we first estimated multiple IBD sharing within each of eight groups separately using SOLAR and then combined all the IBD sharing for further analysis using the variance components method implemented in SOLAR for the combined data.

\section{Results}

Data on height were available from 6752 individuals in 2508 families from the FBPP study. The characteristics of the family members for each group are presented in Table 1.

Table 1 Characteristics of families

\begin{tabular}{|c|c|c|c|c|c|}
\hline \multirow[b]{2}{*}{ Population } & \multirow[b]{2}{*}{$\%$ of male } & \multirow[b]{2}{*}{ Number of height } & \multirow[b]{2}{*}{ Age (years) } & \multicolumn{2}{|c|}{ Height $(C M)$} \\
\hline & & & & Female & Male \\
\hline GenNet European American & 47.2 & 598 & $45.0(14.0)$ & $163.2(6.3)$ & $178.1(7.1)$ \\
\hline GenNet African American & 40.3 & 595 & $40.5(11.6)$ & $164.1(7.2)$ & $176.8(7.1)$ \\
\hline GENOA European American & 47.5 & 749 & $56.3(9.8)$ & $162.4(6.0)$ & $176.7(6.6)$ \\
\hline GENOA African American & 34.5 & 611 & $56.5(9.4)$ & $164.8(6.2)$ & $177.7(6.3)$ \\
\hline GENOA Mexican American & 38.8 & 778 & $55.8(11.6)$ & $157.9(5.6)$ & $170.6(7.0)$ \\
\hline HyperGEN European American & 47.5 & 1100 & $60.9(8.7)$ & $161.2(6.2)$ & $174.9(6.3)$ \\
\hline HyperGEN African American & 30.8 & 1252 & $50.7(11.0)$ & $162.5(6.1)$ & $175.6(6.8)$ \\
\hline SAPPHIRe Asian & 45.3 & 1069 & $54.0(12.2)$ & $155.9(5.2)$ & $167.5(6.0)$ \\
\hline Combined & 41.4 & 6752 & $53.6(12.3)$ & $161.3(6.7)$ & $174.5(7.5)$ \\
\hline
\end{tabular}


Table 2 Results of linkage analysis of individual genome scans

\begin{tabular}{clcccc}
\hline Chromosome & Marker & Location & Region & LOD & Study \\
\hline 1 & D1S1631 & 136 & $115-143$ & 2.25 & GENOA African American \\
3 & D3S1763 & 175 & $160-181$ & 2.06 & GENOA European American \\
5 & D5S816 & 137 & $127-178$ & 2.26 & HyperGen European American \\
& D5S816 & 134 & $123-148$ & 1.48 & SAPPHIRe Asian \\
6 & D6S1053 & 78 & $62-94$ & 2.66 & GENOA European American \\
14 & D14S592 & 67 & $58-92$ & 3.67 & GENOA European American \\
& D14S592 & 67 & $48-96$ & 1.60 & SAPPHIRe Asian \\
\hline
\end{tabular}

The height for females (161.3 cM) and males (174.5) was significantly different $(P<0.0001)$ from each other in the combined sample. The number of families in each group is from 605 to 1410 and the total number of families is 2508 families in the combined data. The mean sibship size in each group is from 1.7 to 3.6 , and the mean sibship size is 2.8 in the combined data. The heritability of height for the eight groups varied from 0.75 to 0.98 , which is compatible with previous reports. ${ }^{4-6}$ For individual genome scans, the region showing the strongest evidence for linkage was found in GENOA European Americans, centered at $14 q 21.1(\mathrm{MLS}=3.67$ at marker D14S592 ) (Table 2). The same region also showed evidence of linkage in SAPPHIRe Asians with a $L O D=1.60$. To obtain the genomewide empirical $P$-value, we performed 250 simulations. We obtained MLS > 3.67 19 times, giving an empirical genomewide $P$-value of 0.08 . Four other regions with a $\operatorname{LOD}>2.0$ (nominal $P=0.0012$ ) were found in individual genome scans. These regions are at $1 p 11,3 q 25,5 q 23$, and $6 q 13$ (Table 2).

The genome scan result for the combined IBD analysis is presented in Figure 1. Seven regions with LOD $>1$ (nominal $P=0.016)$ are also listed in Table 3 as demonstrating suggestive evidence for linkage. The strongest support for linkage was found at $7 \mathrm{q} 36.1$ region $(174 \mathrm{~cm}$, marker D7S3058) with multipoint $\mathrm{LOD}=2.46$.

\section{Discussion}

Hirschhorn et $a l^{4}$ and Perola et $a l^{5}$ reported strong evidence for linkage on chromosome 7 (150 cM, marker D7S2195, $\mathrm{LOD}=3$. 40 ; and marker $164 \mathrm{~cm}, \mathrm{D} 7 \mathrm{~S} 1826, \mathrm{LOD}=2.91)$. Encouragingly, the most significant result in our combined analysis was also found around the same region $(174 \mathrm{~cm}$, marker D7S3058, LOD $=2.46$ ), which makes it likely a true positive result.

Another two regions showing suggestive evidence in our study have been reported previously. Our chromosome 2 region $(2 \mathrm{p} 12,92 \mathrm{~cm}$, marker $\mathrm{D} 2 \mathrm{~S} 1394, \mathrm{LOD}=1.27)$ has been reported by Hirschhorn et al ${ }^{4}(104 \mathrm{~cm}$, marker D2S113, $\mathrm{LOD}=2.23)$. Thompson et $a l^{12}$ have reported linkage on chromosome 20 (20p11, $34.22 \mathrm{~cm}$, marker D20S66,

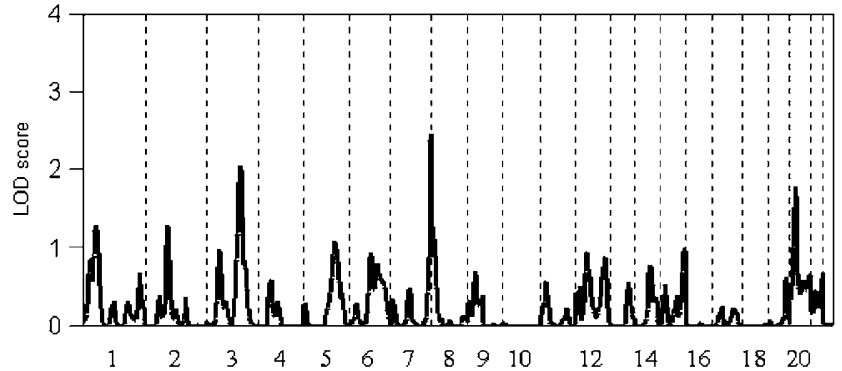

Figure 1 Genome-scan results for combined linkage analysis. The $X$-axis is the chromosome location and the $Y$-axis is the LOD score. Each chromosome is scaled according to its length from the genetic map.

Table 3 Evidence of linkage analysis in combined samples

\begin{tabular}{llrll}
\hline Chromosome & Marker & Location & Region & LOD \\
\hline 1 & D1S1622 & 56 & $48-63$ & 1.27 \\
2 & D2S1394 & 92 & $87-99$ & 1.27 \\
3 & D3S1764 & 146 & $134-158$ & 2.03 \\
5 & D5S1505 & 135 & $131-138$ & 1.08 \\
7 & D7S3058 & 174 & $167-182$ & 2.46 \\
8 & D8S277 & 5 & $1-15$ & 1.26 \\
20 & D20S604 & 29 & $20-35$ & 1.77 \\
\hline
\end{tabular}

LOD $=3.0$ ). Although none of the linkage studies reported previously replicated this finding, we did find suggestive evidence at this region $(29 \mathrm{~cm}$, marker D20S604, LOD $=1.77$ ).

The strongest finding in all the individual studies was on $14 q 21.1$ (MLS $=3.67$ ) with a genomewide empirical $P$-value of 0.08 , which is not significant at the $\alpha=0.05$ level. None of the linkage studies reported previously has shown evidence for linkage at this region. More evidence from future studies is needed to clarify this result.

It has been shown by simulation studies that the power to detect a contributing locus with a small effect in a genome-scan study with moderate sample size is very 
limited when an acceptable type I error rate is maintained. ${ }^{4}$ In a recent review paper, Altmuller et al $^{13}$ compared 31 whole-genome scans for different human complex diseases and found that the most obvious difference influencing success in finding linkage across studies was sample size. Pooling data from multiple studies will greatly increase power assuming the variations among studies are due to random sampling error. Pooling data also will reduce the type I error rates resulting from many independent tests of common hypotheses. By performing linkage analysis in our combined data, we found suggestive evidence for linkage at three regions reported previously for linkage with adult height.

Power calculations for this study have been undertaken under a simplified scenario using a method developed by Sham et $a l^{14}$ For a diallelic QTL with equal allele frequency, we assumed a model with 5\% QTL additive variance, 70\% residual shared variance and a recombination rate between marker and QTL of 5\%. Considering a sample size of 6700 with sibship size of 3 with, the power to achieve LOD $=3$ is $97 \%$. If the sibship size decreases to 2 , the power will decrease to only $25 \%$ in the same sample. If we increase QTL additive variance from 5 to $10 \%$ and keep other parameters the same, the power to detect the QTL with LOD $=3$ will increase to $97 \%$ even with sibship size of 2 . Although the average sibship size of the FBPP study is close to 3 (2.8), the variation of pedigree structure among different networks could decrease the power under an ideal situation. Based on the above discussion, it is reasonable to expect a high power to detect a QTL with an effect size of $10 \%$ in the combined sample.

No obvious candidate gene for adult height was found for the chromosome 7 region (7q31) in an initial search in NCBI databases. For the chromosome 2 region (2p12), a candidate gene is bone morphogenetic protein 10 (BMP10). This gene is a member of TGF- $\beta$ family of growth factors. For the region at chromosome 20 (20p11), an obvious candidate gene is BMP2. Like BMP10, BMP2 also belongs to the TGF- $\beta$ family and induces bone formation. In an association study by Thompson et al, ${ }^{12}$ no significant association was found with BMP2 in a subset of 20 tallest and 20 shortest individuals selected from a sample of over 500 Pima Indians. However, the power to detect any association is low with such a small sample size.

In summary, by combining the data sets from eight studies in the FBPP, we replicated evidence for a QTL influencing adult height in chromosome 7 (7q31) $(\mathrm{LOD}=2.46)$, which has been reported in two studies previously. Suggestive linkage (LOD $>1$ ) was found in another six regions in our combined analysis. Evidence for linkage for two of these regions $(2 \mathrm{p} 12,20 \mathrm{p} 11)$ has also been reported previously.

\section{Acknowledgements}

We thank Thomas Dyer from Southwest Foundation for Biomedical Research, San Antonio, TX, USA for his helpful suggestions using SOLAR package. We are grateful to two anonymous reviewers whose constructive comments were very helpful in preparing the paper for publication. We thank Christy Chang of Johns Hopkins University for her discussion of data cleaning. This work was supported by grants from the National Heart, Lung and Blood Institute (UO1 HL54485; HL54466; HL65702; HL54481; HL45508; HL47910; HL51021; HL54464; HL54473; HL54496; HL54472; HL54515; HL54495; HL54471; HL54509; 2HHZ598; HL65702; HL54527).

\section{References}

1 Carmichael CM, McGue MA: Cross-sectional examination of height, weight and body mass index in adult twins. J Gerontol A: Biol Sci Med Sci 1995; 50: B237-B244.

2 Preece MA: The genetic contribution to stature. Horm Res 1996; 45: $56-58$.

3 Silventoinen K, Kaprio J, Lahelma E, Loskenvuo M: Relative effect of genetic and environmental factors on body height: differences across birth cohorts among Finnish men and women. Am J Public Health 2000; 90: 627-630.

4 Hirschhorn JN, Lindgren CM, Daly MJ et al: Genomewide linkage analysis of stature in multiple populations reveals several regions with evidence of linkage to adult height. Am J Hum Genet 2001; 69: 106-116.

5 Perola M, Ohman M, Hiekkalinna T et al: Quantitative-trait-locus analysis of body-mass index and of stature, by combined analysis of genome scans of five Finnish study groups. Am J Hum Genet 2001; 69: 117-123.

6 Wiltshire S, Frayling TM, Hattersley AT et al: Evidence for linkage of stature to chromosome $3 \mathrm{p} 26$ in a large U.K. Family data set ascertained for type 2 diabetes. Am J Hum Genet 2002; 70: 543-546.

$7 \mathrm{Xu}$ J, Bleecker ER, Jongepier $\mathrm{H}$ et al: Major recessive gene(s) with considerable residual polygenic effect regulating adult height: Confirmation of genomewide scan results for chromosomes 6, 9, and 12. Am J Hum Genet 2002; 71: 646-650.

8 Allison DB, Heo M: Meta-analysis of linkage data under worstcase conditions: a demonstration using the human $\mathrm{OB}$ region. Genetics 1998; 148: 859-865.

9 Palmer LJ, Barnes KC, Burton PR et al: Meta-analysis for linkage to asthma and atopy in the chromosome 5q31-33 candidate region. Hum Mol Genet 2001; 10: 891-899.

10 The FBPP Investigators. A multi-center genetic study of hypertension. The Family Blood Pressure Program (FBPP). Hypertension 2002; 39: 3-9.

11 Almasy L, Blangero J: Multipoint quantitative trait linkage analysis in general pedigrees. Am J Hum Genet 1998; 62: 1198-1211.

12 Thompson DB, Ossowski V, Janssen RC, Knowler WC, Bogardus C: Linkage between stature and a region on chromosome 20 and analysis of a candidate gene, bone morphogenetic protein 2. Am J Med Genet 1995; 59: 495-500.

13 Altmuller J, Palmer LJ, Fischer G, Scherb H, Wjst M: Genomewide scans of complex human diseases: true linkage is hard to find. $A m$ J Hum Genet 2001; 69: 936-950.

14 Sham PC, Cherny SS, Purcell S, Hewitt JK: Power of linkage versus association analysis of quantitative traits, by use of variancecomponents models, for sibship data. Am J Hum Genet 2000; 66: $1616-1630$. 